\title{
The RNA interference pathway affects midgut infection- and escape barriers for Sindbis virus in Aedes aegypti
}

\author{
Cynthia CH Khoo, Joseph Piper, Irma Sanchez-Vargas, Ken E Olson and Alexander WE Franz*
}

\begin{abstract}
Background: The RNA interference (RNAi) pathway acts as an innate antiviral immune response in Aedes aegypti, modulating arbovirus infection of mosquitoes. Sindbis virus (SINV; family: Togaviridae, genus: Alphavirus) is an arbovirus that infects Ae. aegypti in the laboratory. SINV strain TR339 encounters a midgut escape barrier (MEB) during infection of Ae. aegypti. The nature of this barrier is not well understood. To investigate the role of the midgut as the central organ determining vector competence for arboviruses, we generated transgenic mosquitoes in which the RNAi pathway was impaired in midgut tissue of bloodfed females. We used these mosquitoes to reveal effects of RNAi impairment in the midgut on SINV replication, midgut infection and dissemination efficiencies, and mosquito longevity.
\end{abstract}

Results: As a novel tool for studying arbovirus-mosquito interactions, we engineered a transgenic mosquito line with an impaired RNAi pathway in the midgut of bloodfed females by silencing expression of the Aa-dcr2 gene. In midgut tissue of the transgenic Carb/dcr16 line, Aa-dcr2 expression was reduced $\sim 50 \%$ between 1-7 days post-bloodmeal (pbm) when compared to the recipient mosquito strain. After infection with SINV-TR339EGFP, Aa-dcr2 expression levels were enhanced in both mosquito strains. In the RNAi pathway impaired mosquito strain SINV titers and midgut infection rates were significantly higher at 7 days pbm. There was also a strong tendency for increased virus dissemination rates among the transgenic mosquitoes. Between 7-14 days pbm, SINV was diminished in midgut tissue of the transgenic mosquitoes. Transgenic impairment of the RNAi pathway and/or SINV infection did not affect longevity of the mosquitoes.

Conclusions: We showed that RNAi impaired transgenic mosquitoes are a useful tool for studying arbovirus-mosquito interactions at the molecular level. Following ingestion by Ae. aegypti, the recombinant SINV-TR339EGFP was confronted with both MEB and a midgut infection barrier (MIB). Impairment of the RNAi pathway in the midgut strongly reduced both midgut barriers for the virus. This confirms that the endogenous RNAi pathway of Ae. aegypti modulates vector competence for SINV in the midgut. The RNAi pathway acts as a gatekeeper to the incoming virus by affecting infection rate of the midgut, intensity of infection, and dissemination from the midgut to secondary tissues.

\section{Background}

The RNA interference (RNAi) pathway is an innate immune pathway of invertebrates such as nematodes, trypanosomes, hydra, planaria, and insects [1]. In mosquitoes, the RNAi pathway has been shown to act as an antiviral immune pathway that is able to effectively modulate the replication pattern of arthropod-borne viruses

\footnotetext{
*Correspondence: afranz@colostate.edu

${ }^{1}$ Arthropod-Borne and Infectious Diseases Laboratory, Department of Microbiology, Immunology and Pathology, Colorado State University, Fort Collins, CO 80523 USA

Full list of author information is available at the end of the article
}

(arboviruses) [2-6]. It has been postulated that RNAi functions as a gatekeeper in mosquitoes, modulating arbovirus replication to allow virus transmission but preventing virus concentrations that could lead to fitness costs and pathogenic effects [6]. Consequently, RNAi is potentially a major factor determining the vector competence of mosquitoes for arboviruses.

Sindbis virus (SINV; family: Togaviridae; genus: Alphavirus) is an arbovirus with a positive sense singlestranded RNA genome. A dsRNA intermediate is formed during replication, which triggers the RNAi pathway causing homology-dependent destruction of viral RNA 
[3]. Since SINV is able to establish persistent infections in the mosquito, the virus must have developed strategies to cope with the antiviral RNAi pathway in the insect host. Potential RNAi evasion strategies for alphaviruses are active suppression of the RNAi pathway and - similar to flaviviruses - sequestration of the dsRNA replicative intermediate within cellular membrane structures [7]. Under natural conditions, SINV circulates between Culex sp. and birds with humans acting as dead end hosts [8]. However, in the laboratory the virus is transmissible by the well characterized mosquito vector Aedes aegypti, prompting researchers to use the SINV-Ae. aegypti combination as a model to study arbovirus-mosquito interactions at the molecular level. After ingestion of a viremic bloodmeal by a competent mosquito, SINV enters midgut epithelial cells and begins replicating [9]. From the midgut the virus disseminates to secondary tissues such as muscles surrounding the alimentary tract, fat body, hemocytes, nerve tissue, and finally salivary glands. Once SINV enters the saliva, the virus has completed its extrinsic incubation period and the mosquito is able to transmit the virus to a new host [9]. The TR339 strain of SINV is based on a consensus sequence derived from the type strain AR339 that has been isolated in Egypt [10-12]. For this study, we used a full-length infectious cDNA clone of the virus with the enhanced green fluorescent protein (EGFP) marker gene inserted downstream of a second subgenomic promoter [3]. After ingestion by females of the Ae. aegypti RexD strain, SINV-TR339 has been shown to encounter an escape barrier in the midgut (MEB); whereas reported midgut infection rates were $>90 \%$, dissemination rates only reached $40 \%[9,13]$.

Midgut infection barrier (MIB) and/or MEB have been observed for a number of other alphaviruses and for flaviviruses [14,15]. MIB prevents ingested arboviruses from entering and replicating in mesenteronal (midgut) cells, whereas MEB prevents virions from escaping from the basal lamina of midgut cells and disseminating to other tissues in the hemocoel. Often these barriers depend on the amount of virus ingested by the mosquito because the virus has to reach a certain threshold to either establish an infection in the midgut or to disseminate to other tissues $[9,14-16]$. Furthermore, dose-independent MIB or MEB have been reported, implying an incompatibility between arbovirus and vector at the midgut level, thus preventing arboviruses from entering or exiting the epithelial cells [13,17-20]. Until now, the molecular nature of MIB and MEB, which appears to depend on specific virus-mosquito strain combinations, is not well understood. However, recent correlation analysis of RNAi pathway genes with MIB and MEB combined with linkage mapping of Aa-dcr2, Aa-r2d2, and Aa-ago2 genes in the genome of Ae. aegypti suggests that MIB and MEB for dengue virus could be RNAi associated phenomena [21].
To investigate the nature of MIB and MEB for SINVTR339EGFP in Ae. aegypti, we impaired the RNAi pathway in the mosquito midgut at a time point when the ingested virus is replicating in cells of the midgut epithelium. We expected that impairment of the RNAi pathway in the midgut of Ae. aegypti would allow the virus to overcome potential MIB and/or MEB and to increase its overall titer in the insect. We chose a transgenic approach to impair the RNAi pathway in the midgut of Ae. aegypti by generating mosquitoes expressing an inverted-repeat (IR) RNA derived from the RNAi pathway gene $\mathrm{Aa}-\mathrm{dcr} 2$ under control of the bloodmeal inducible, midgut-specific Ae. aegypti carboxypeptidase A (AeCPA) promoter [22-25]. According to our strategy the midgut-specific IR effector would produce dsRNA in bloodfed females, triggering RNAi against $A a-d c r 2$ and eventually causing depletion of dicer2 protein in the midgut. This would cause impairment of the RNAi pathway in this tissue. Previously, it has been demonstrated that homologydependent silencing of $d c r 2$ can impair the RNAi mechanism in insect cells and in Ae. aegypti [26-28].

The objectives of this study are to generate transgenic Ae. aegypti mosquitoes with an impaired RNAi pathway in midgut tissue after ingestion of a bloodmeal, to assess vector competence of the transgenic mosquitoes for SINV-TR339EGFP with respect to possible effects on MIB and MEB, and to evaluate if midgut-specific impairment of the RNAi pathway reduces the survival rate of SINV-infected mosquitoes.

\section{Results}

Generation of transgenic Ae. aegypti expressing an IR RNA targeting Aa-dcr2 mRNA

We designed a donor plasmid based on the Mariner Mos1 transposable element (TE) containing an Aa-dcr2 IR expression cassette under control of the bloodmeal inducible, midgut-specific AeCPA promoter (Fig. 1A). The donor plasmid was co-injected with a helper plasmid expressing the Mos1 transposase [29] into 1780 pre-blastoderm embryos of the Ae. aegypti HWE strain. The survival rate was $10.3 \%$. After outcrossing to the HWE recipient strain, $115 \mathrm{G}_{0}$ families were established and their offspring $\left(G_{1}\right)$ were screened for eye-specific EGFP expression. We selected 10 different mosquito families that produced transgenic offspring, Carb/dcr16, 29, 44, $54,69,79,113,125,126$, and 146 .

\section{Levels of Aa-dcr2 silencing among the transgenic Ae. aegypti lines}

As an initial molecular characterization we analyzed $A a$ $d c r 2$ mRNA expression in midguts of nine of the 10 transgenic lines after bloodfeeding by quantitative reverse transcriptase PCR (qRT-PCR). Line Carb/dcr69 was eventually lost during mosquito rearing. One week post- 

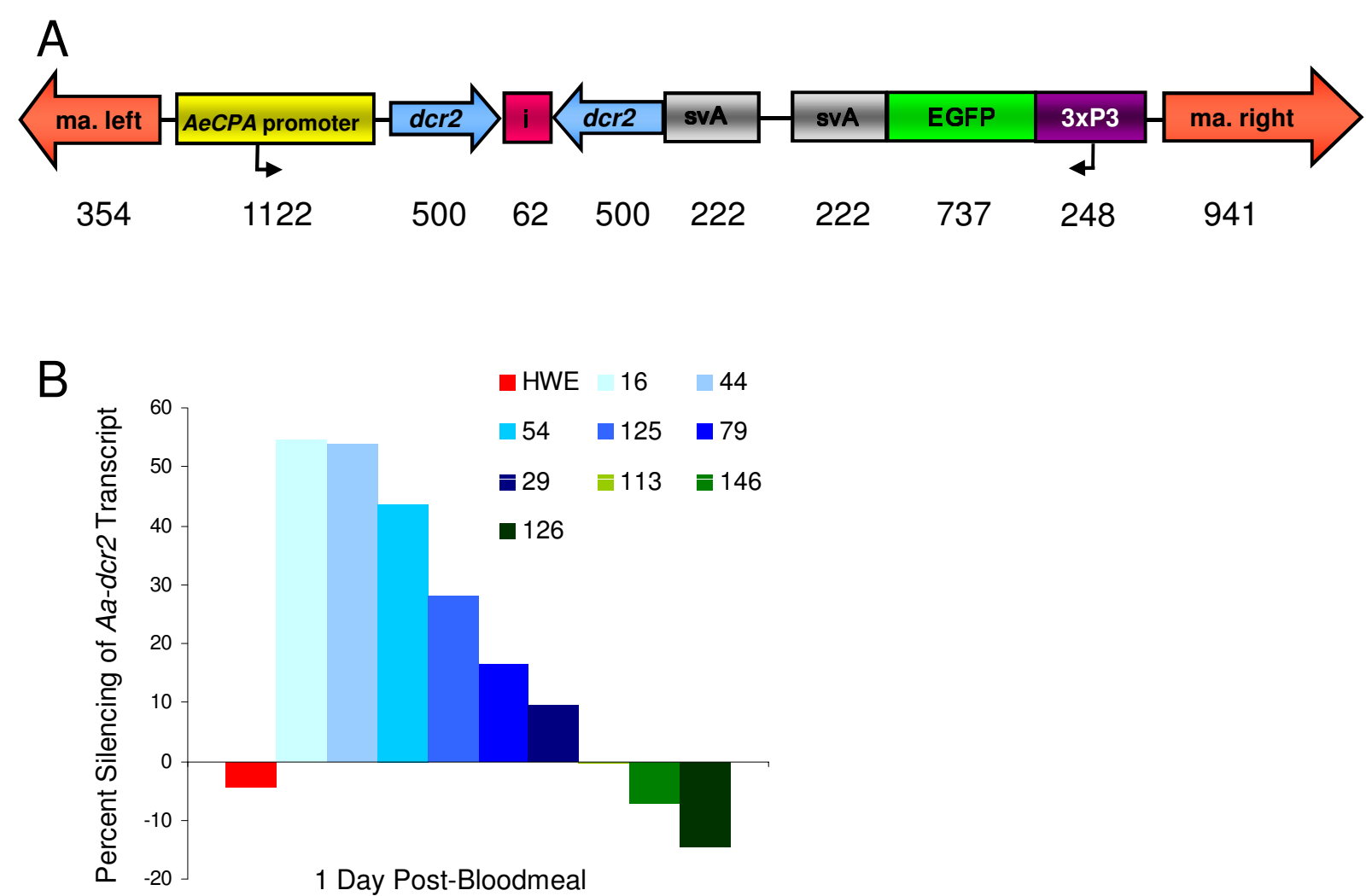

Figure 1 Transgene design to silence Aa-dcr2 in the midgut of bloodfed females and molecular characterization of transgenic mosquito lines. A) Five hundred base-pair (bp) cDNAs in sense and anti-sense orientations corresponding to a portion of Aa-dcr2 were used for the inverted repeat (IR) construction. Sense and anti-sense cDNA fragments of Aa-dcr2 were separated by the small intron of the Aa-sialonkinin / gene and placed downstream of the Aa-carboxypeptidase A promoter. A transcription termination signal derived from SV40 was added downstream of the IR construct. Numbers below the diagram indicate sizes in bp. Abbreviations: ma. left, ma. right = left, right arms of the Mos 1 Mariner transposable element (TE); AeCPA promoter = promoter region of the Ae. aegypti carboxypeptidase $A$ gene; $d c r 2=c D N A$ fragments corresponding to the Aa-dcr2 gene; $i=$ minor intron of the Ae. aegypti sialokinin / gene; svA = transcription termination signal derived from the SV40 virus; EGFP = green fluorescent protein marker; 3xP3 = eye tissue-specific promoter. B) Percentage of midgut-specific silencing of Aa-dcr2 mRNA among nine different transgenic Ae. aegypti lines at 1 day pbm. Aa-dcr2 expression levels in midguts of bloodfed females were normalized for gene expression levels of sugarfed females of the lines at the same time point. Bloodmeals were obtained from mice. Each sample consisted of total RNA from a pool of 20 midguts.

emergence females of the nine lines were bloodfed on mice. Relative $A a-d c r 2$ mRNA accumulation was reduced by $>50 \%$ in mosquito midguts of lines Carb/dcr16 and Carb/dcr44 at day 1 post-bloodmeal (pbm) as compared to sugarfed control mosquitoes (Fig. 1B). For lines Carb/ dcr54, 125, 79, and 29, relative levels of Aa-dcr2 mRNA reduction were between $10-45 \%$. On the contrary, for lines Carb/dcr126, 146, and the non-transgenic HWE control relative $A a-d c r 2$ mRNA levels were increased in mosquito midguts. Based on the Aa-dcr2 mRNA expression profile of Carb/dcr16 females, we selected this line for further vector competence studies with SINVTR339EGFP.

\section{Characterization of the transgene integration site in Carb/ dcr16 mosquitoes}

The transgene integration site in the genome of Carb/ dcr16 mosquitoes was defined by Genome Walking. We confirmed the stable integration of the Mos1 based transgene into the genome of HWE mosquitoes by the fact that DNA sequences flanking the left and right arms of the TE were continuous (Fig. 2A). The TE integration site is in a non-protein encoding region at nucleotide position 858,262 of contig 503, supercontig 1.6. Absence of any other sequences from the Genome Walking libraries strongly suggests that integration of the TE occurred as a single copy.

\section{Phenotypic analysis of SINV-TR339EGFP}

The 720 base-pair coding sequence of the EGFP gene was inserted into a recombinant cDNA clone of SINV-TR339. The marker gene was placed under control of the engineered, duplicated subgenomic promoter that was located upstream of the sequence encoding the viral structural genes. Growth curve analysis of SINVTR339EGFP in Vero cells revealed an increase in virus 
A

nt 858,201

Mos1 right arm

ACCCTATTACAACGCTTGTTCGTCAGTTCTATCGCTGCCGTCGTCGAGAACGGACGCAAGTATCAGGTGTACAAG....

....ACTTGTACACCTGGTATTGTGTTCCCCCTTGATCGGTTTCTCGTTATTGTCAGAAGAGTTTCCAAGCGCGCTTTG

Mos1 left arm

nt 858,321

Integration Site: supercontig 1.6; contig 503; nt 858,262

B

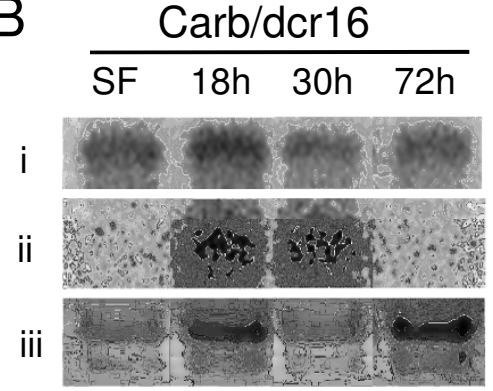

HWE

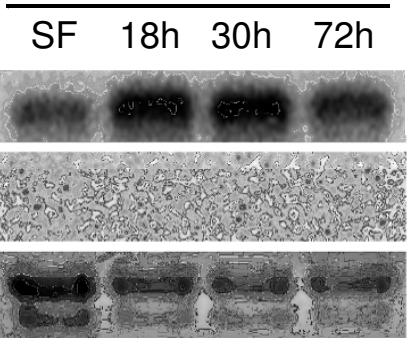

Aa-dcr2 mRNA ( 6000 nt)

Aa-dcr2 IR RNA ( 500 nt)

rRNA (loading control)
C

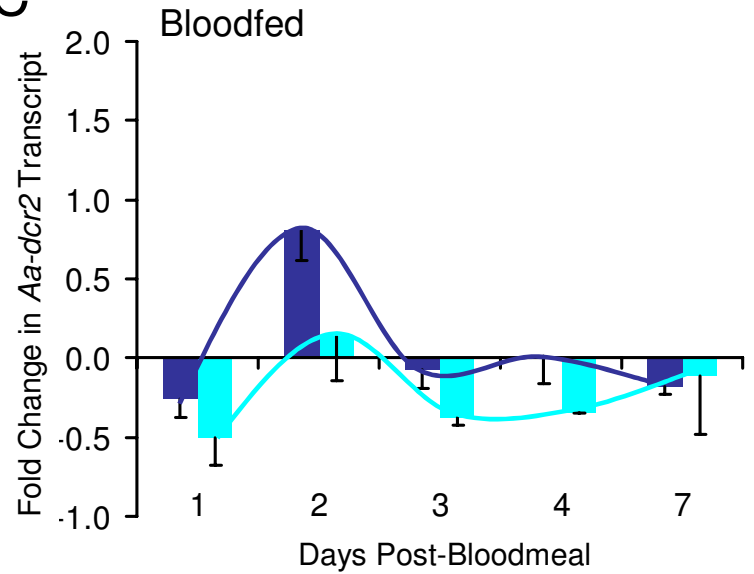

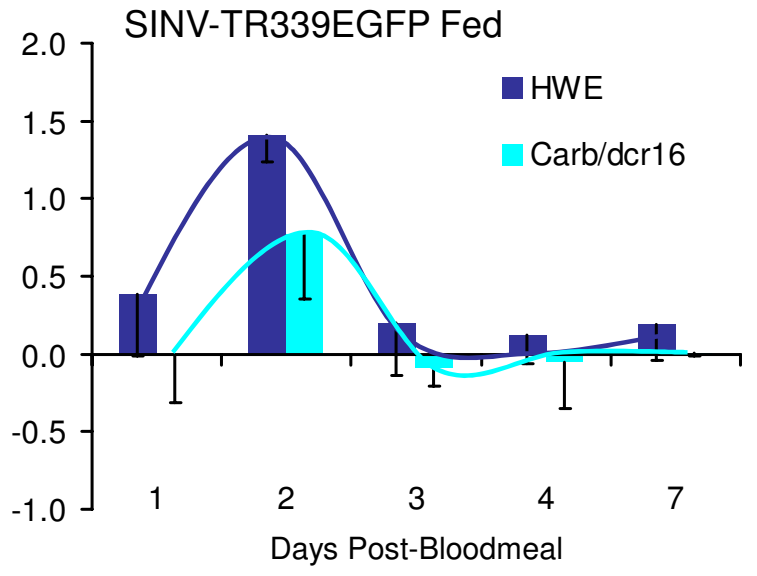

Figure 2 Molecular characterization of Carb/dcr16 mosquitoes. A) Genomic DNA sequences flanking the left and right arms of the modified Mariner Mos 1 TE after its integration into the genome of Carb/dcr16 mosquitoes. In bold: duplicated endogenous Mos 1 target site; green letters: partial DNA sequence of the right arm of the Mos 1 TE; blue letters: partial DNA sequence of the left arm of the Mos 1 TE. B) Northern blot analysis of Aa-dcr2 mRNA and transgene expression levels in midguts of Carb/dcr16 and HWE control females at 18, 30, and $72 \mathrm{~h}$ pbm (SF $=$ midgut RNA of sugarfed females). C) Levels of midgut-specific Aa-dcr2 silencing among bloodfed or SINV-TR339EGFP infected Carb/dcr16 and HWE females at 1-7 days pbm. Aa-dcr2 expression levels in midguts of bloodfed females were normalized for gene expression levels of sugarfed females at similar time points. Mosquitoes obtained artificial bloodmeals consisting of defibrinated sheep blood. Values below zero indicate silencing of Aa-dcr2 and values above zero indicate up-regulation of the gene. Wave-shaped lines represent the Aa-dcr2 expression profiles in midguts of Carb/dcr16 and HWE females. Bars represent mean values of three replicates for HWE and two replicates for Carb/dcr16 mosquitoes. Each replicate consisted of total RNA from a pool of 20 midguts (error bars $=$ SEM).

titer from $1 \times 10^{6}$ to $4 \times 10^{7} \mathrm{pfu} / \mathrm{ml}$ between 15 and $38 \mathrm{~h}$ post-infection (multiplicity of infection: 0.01 ). Then the titer gradually decreased to $2 \times 10^{6}$ at 65 h post-infection. The pattern of the growth curve was similar to that observed for the TR339 strain of SINV lacking a duplicated subgenomic promoter [13]. Furthermore, strong EGFP expression was observed among the cells at $38 \mathrm{~h}$ post-infection. However, in SINV-TR339EGFP infected tissue such as the mosquito midgut, EGFP expression was often rather low even though virus titers proved to be relatively high (data not shown). This observed discrepancy between viral marker gene expression and actual titers prompted us in the following experiments to base SINVTR339EGFP detection in mosquitoes on intensity of infection rather than visualization of EGFP expression. 


\section{Evaluation of transgene expression and Aa-dcr2 mRNA levels in midguts of Carb/dcr 16 females}

Detection of a single RNA band corresponding to a size of $\sim 500 \mathrm{nt}$ by Northern blot analysis showed that $A a-d c r 2$ derived IR RNA was transcribed in midguts of Carb/ dcr16 females 18-30 h after receiving a non-infectious bloodmeal (Fig. 2Bii). A similar signal was not detected at a later time point or in midguts of sugarfed Carb/dcr16 females and in the HWE control. This temporal and spatial expression pattern was in agreement with those observed for other transgenes controlled by the AeCPA promoter [23,24]. Hybridization signal intensities for $A a$ dcr2 mRNA among midgut RNA of bloodfed Carb/dcr16 mosquitoes were considerably weaker at $18-72 \mathrm{~h} \mathrm{pbm}$ compared to those of bloodfed HWE at similar time points (Fig. 2Bi). This indicates silencing of the RNAi pathway gene in midguts of the bloodfed transgenic mosquitoes. In addition, we assessed the Aa-dcr2 mRNA expression profile for Carb/dcr16 mosquitoes during one week by qRT-PCR. Aa-dcr 2 expression in midguts of bloodfed females followed a wave-like pattern with lowest expression in the transgenic line at days 1,3 and 4 pbm and maximal expression at day 2 pbm (Fig. 2C). Accumulation of $A a-d c r 2$ mRNA was reduced in midguts of Carb/dcr16 females as compared to the HWE control with the exception of day $7 \mathrm{pbm}$, a time point when the transgene was no longer expressed. We observed that $A a-$ $d c r 2$ expression profiles were generally less elevated in Carb/dcr16 and HWE mosquitoes that had received an artificial bloodmeal containing defibrinated sheep blood than in mosquitoes that had been allowed to feed on mice (data not shown). After ingestion of a bloodmeal containing SINV-TR339EGFP (titer in the bloodmeal: $2.2 \times 10^{7}$ $\mathrm{pfu} / \mathrm{ml}), A a-d c r 2 \mathrm{mRNA}$ levels in midguts of Carb/dcr16 and HWE followed a similar wave-like pattern. Aa-dcr2 mRNA accumulation was substantially increased in Carb/dcr16 and HWE mosquitoes during the one week observation even though the overall level of $A a-d c r 2$ mRNA was still lower in the transgenic females than in HWE. This suggests that replicating SINV-TR339EGFP has triggered the RNAi pathway in the mosquito midgut.

\section{Effects of Aa-dcr2 silencing in the midgut of Carb/dcr16 females on intensity of SINV-TR339EGFP infection, infection rate, and dissemination in an initial experiment}

To test whether midgut-specific silencing of $A a-d c r 2$ affects the vector competence for SINV-TR339EGFP, infection intensities and virus infection and dissemination rates were evaluated in Carb/dcr16 mosquitoes. In an initial experiment (virus titer in the bloodmeal: $1.8 \times$ $10^{7} \mathrm{pfu} / \mathrm{ml}$ ), midgut infection rate and intensity of virus infection were significantly higher in Carb/dcr16 than in HWE mosquitoes at 7 days pbm (Fig. 3A). We observed that $21 / 30 \mathrm{Carb} / \mathrm{dcr} 16$ females were infected with a 1300-fold higher mean virus titer than the HWE control. In contrast, only $2 / 30 \mathrm{HWE}$ mosquitoes had measurable virus infection in their midguts. Accordingly, 53\% of the remaining mosquito bodies of Carb/dcr16 females were infected with SINV at 7 days pbm, whereas no HWE carcasses showed any detectable infection. This indicates that midgut infection rate and intensity affect the dissemination potential of the virus to secondary tissues. However, at 14 days pbm the overall SINV infection patterns of Carb/dcr16 females were no longer significantly different from those of the HWE control. These results suggest that SINV-TR339EGFP encountered MIB and MEB in HWE mosquitoes at 7 days pbm, whereas in the RNAiimpaired Carb/dcr16 females these barriers were not evident.

\section{Effects of Aa-dcr2 silencing in the midgut of Carb/dcr16 females on mean intensities of SINV-TR339EGFP infection, infection and dissemination rates}

To confirm this observation, we repeated the experiment three more times and assessed mean intensity of SINV infection and midgut infection rates. To reveal mean midgut dissemination rates for the virus, two additional replicates of the experiment were analyzed. SINVTR339EGFP titers in the bloodmeals ranged from 1.7-2.7 $\times 10^{7} \mathrm{pfu} / \mathrm{ml}$. The mean intensity of virus infection in midguts of Carb/dcr16 females (14,000 pfu/ml) was $>8$ fold higher than in the control at 7 days pbm, which was highly significant (Fig. 3B). Similarly, in the remaining mosquito bodies the difference between HWE and Carb/ dcr16 females was statistically significant. In contrast, mean intensities of SINV-TR339EGFP infection did not differ significantly between the transgenic strain and the HWE control at 14 days pbm. At this time point however, virus titers were reduced by $83 \%$ in midguts of Carb/ dcr16 mosquitoes as compared to seven days earlier. This effect was observed only in the RNAi-impaired Carb/ dcr16 mosquitoes. Since SINV titers of carcasses were not increased at 14 days pbm as compared to 7 days pbm, we assume that reduction in the intensity of virus infection in midguts was not caused by virus dissemination to secondary tissues.

The mean midgut infection rate with SINVTR339EGFP was significantly higher among Carb/dcr16 mosquitoes (69\%) than among the HWE control (33\%) at 7 days pbm (Fig. 4A). As the standard error in Fig. 4A predicts, midgut infection rates of the HWE mosquitoes had a relatively high variability between experiments. Clearly, in the RNAi-impaired Carb/dcr16 females the midgut infection rates did not fluctuate as strongly. This suggests that HWE responded more sensitively to changes in virus dose present in bloodmeals of different challenge experiments. At 7 days pbm the mean infection rate of the carcasses was significantly lower among HWE 


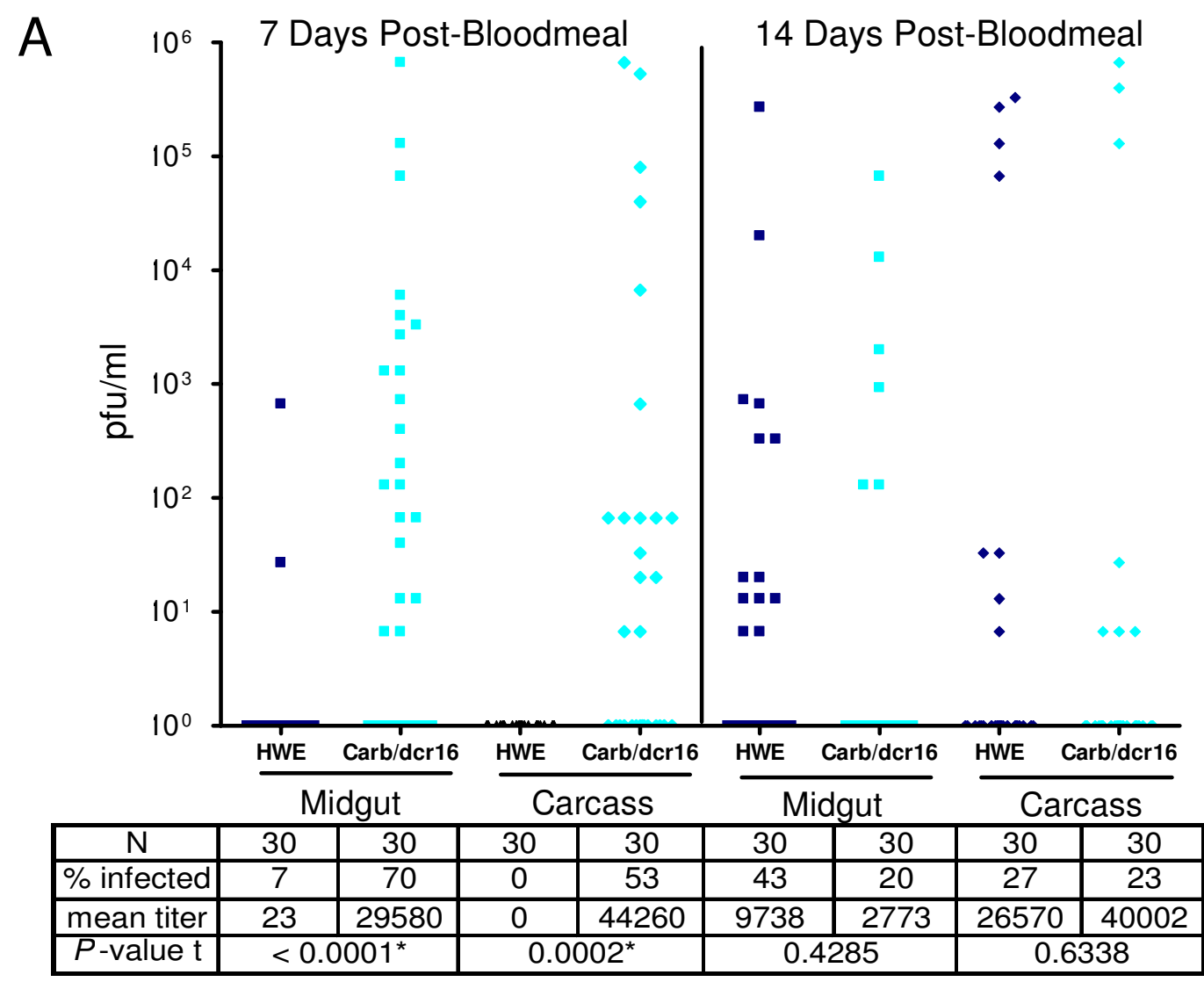

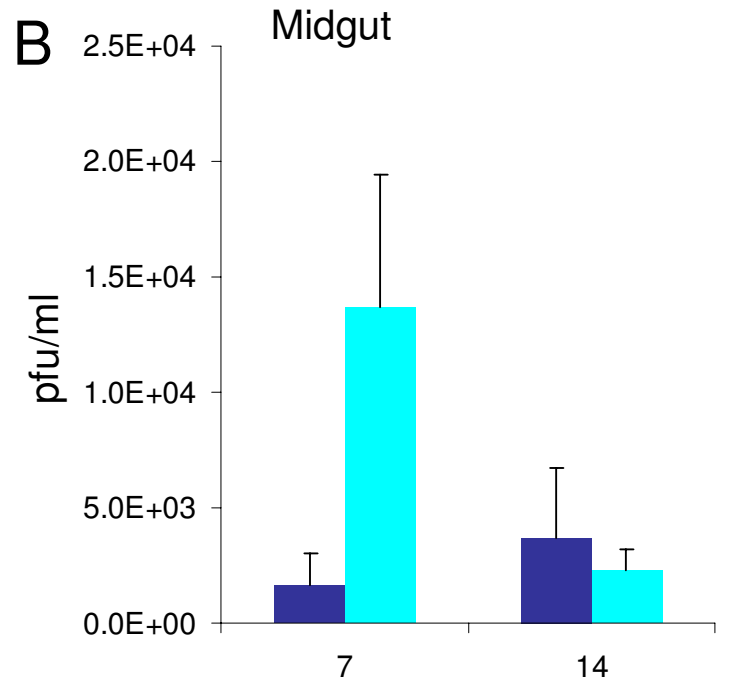

Days Post-Bloodmeal

\begin{tabular}{|c|c|c|c|c|}
\hline $\mathrm{N}$ & 114 & 134 & 114 & 135 \\
\hline$P$-value t & $<0.0001^{*}$ & 0.4288 \\
\hline
\end{tabular}

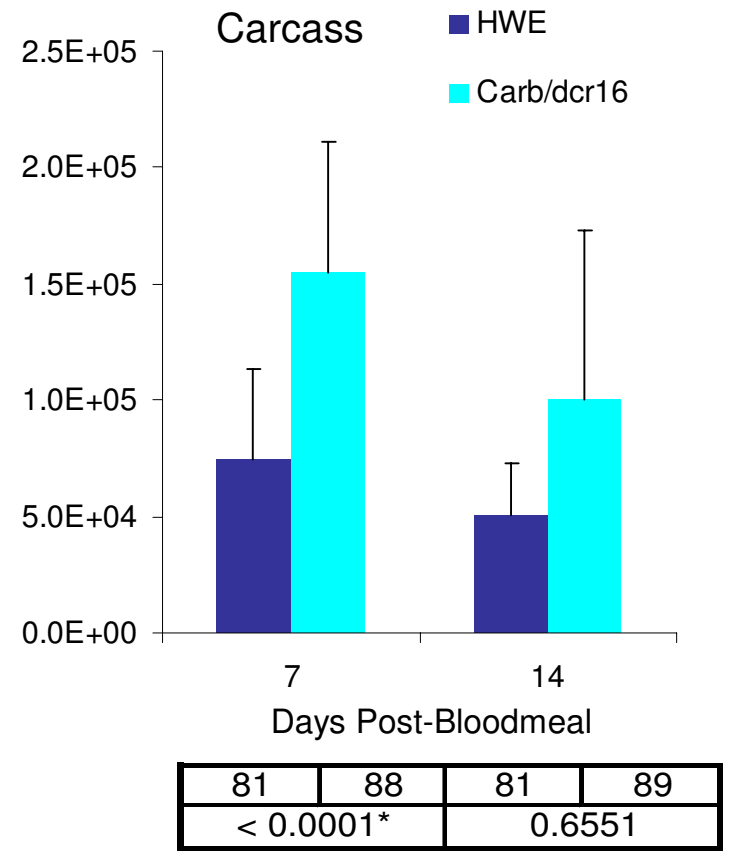

Figure 3 Intensity of SINV-TR339EGFP infection in Carb/dcr16 and HWE mosquitoes. A) Raw data of a single experiment in which Carb/dcr16 females were orally challenged with SINV. Each data point represents the virus titer (pfu/ml) in midgut or carcass of an individual mosquito. $P$-values for intensities of virus infection are shown in the table. B) Mean intensities of SINV infection in midguts and carcasses of Carb/dcr 16 and HWE females at 7 and 14 days pbm. Mean values of three experiments are shown. $\left(\mathrm{N}=\right.$ sample size; ${ }^{*}=$ statistically significantly different $(a=0.05)$; error bars $\left.=\mathrm{SEM}\right)$. 


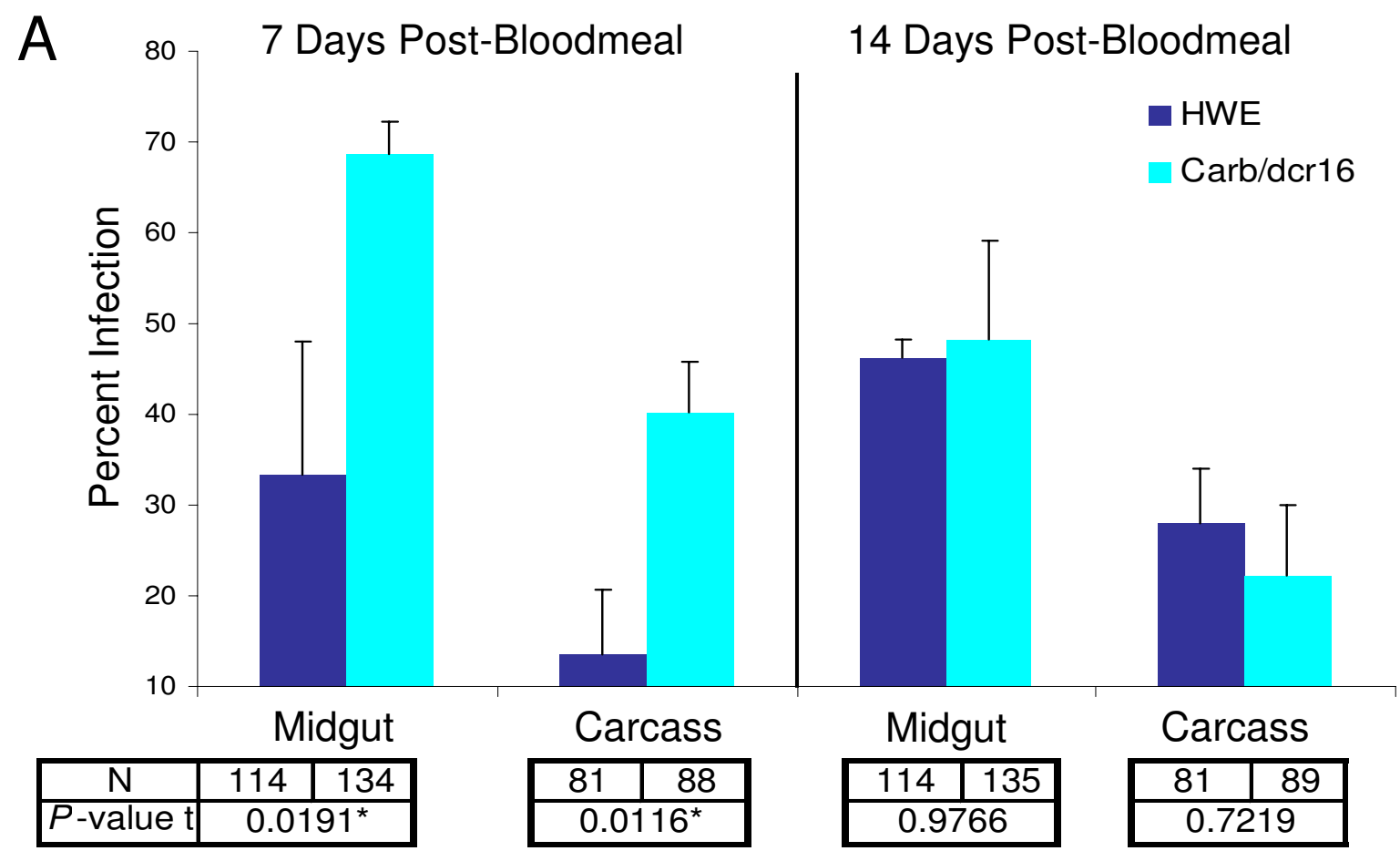

B

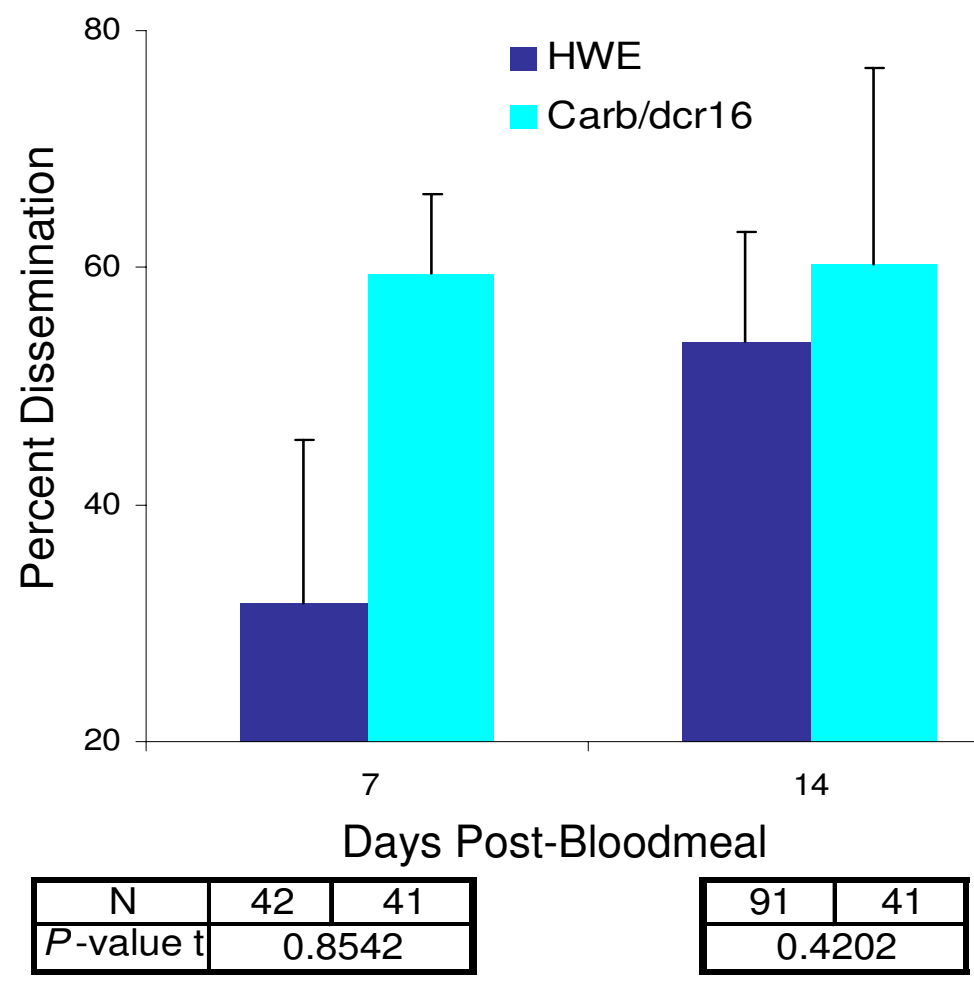

Figure 4 Infection and dissemination rates of SINV-TR339EGFP in Carb/dcr16 and HWE mosquitoes. A) Midgut and carcass infection rates of Carb/dcr 16 and HWE females with SINV at 7 and 14 days pbm. Mean values of three experiments are shown ( $\mathrm{N}=$ sample size; ${ }^{*}=$ statistically significantly different; error bars =SEM). B) Dissemination rate of SINV in Carb/dcr16 and HWE females at 7 and 14 days pbm. Mean values of two experiments are shown ( $\mathrm{N}$ = sample size; error bars = SEM). Infection and dissemination rates were determined by plaque assays. 
than among Carb/dcr16 females. At 14 days pbm mean midgut and carcass infection rates no longer differed significantly between both mosquito strains. In Carb/dcr16 females mean infection rates were decreased by $20 \%$ at 14 days pbm compared to those at 7 days pbm even though in HWE they were increased by $\sim 20 \%$ (Fig. 4A). This is in accordance with the data obtained from the analysis of midgut infection intensity (Fig. 3B), showing that in the transgenic mosquitoes SINV was diminished in midguts after 7 days pbm.

When comparing the mean dissemination rates of SINV-TR339EGFP between HWE and Carb/dcr16, we only considered mosquitoes having infections in both midgut and carcass at 7 or 14 days pbm. In both mosquito strains, virus dissemination rates followed a pattern similar to the midgut infection rates at 7 days pbm (Fig. 4B). Differences were not statistically significant between Carb/dcr16 and HWE mosquitoes even though dissemination rates were about twice as high in Carb/dcr16 females $(60 \%)$ at 7 days pbm. The lack of statistical significance could be due to the smaller sample sizes available for this experiment. However, our data suggest that dissemination rates for SINV-TR339EGFP are dependent on the virus dose ingested by the mosquito.

\section{Survival rates of Carb/dcr16 females after infection with SINV-TR339EGFP}

We evaluated the effect of midgut-specific impairment of the RNAi pathway on the longevity of mosquitoes once they were infected with SINV-TR339EGFP. So far our data have shown that at 7 days pbm the RNAi pathwayimpaired mosquitoes contained higher doses of the virus than the HWE control. We monitored the survival rate of mosquitoes for four weeks after bloodfeeding. Bloodfeeding appeared to have a beneficial effect for both Carb/ dcr16 and HWE females since $50 \%$ of the insects were still alive at day $25 \mathrm{pbm}$ whereas of the sugarfed control only $20 \%$ were alive at the same time point (Fig. 5). When both mosquito strains were infected with SINVTR339EGFP (titer in the bloodmeal: $2.7 \times 10^{7} \mathrm{pfu} / \mathrm{ml}$ ), their longevity was not affected in comparison to noninfected, bloodfed mosquitoes. The survival curves looked similar for Carb/dcr16 and HWE females, indicating that SINV infection did not cause an obvious fitness cost in the RNAi-impaired mosquitoes.

\section{Discussion}

This study demonstrates for the first time a transgenic approach to impair the endogenous RNAi pathway in midgut tissue of Ae. aegypti. Following the principle of activating the RNAi pathway in specific tissues during digestion of a bloodmeal $[24,25,30]$, we generated mosquitoes expressing an $A a-d c r 2$ targeting IR RNA in the midgut to trigger the RNAi pathway against itself. Thus,

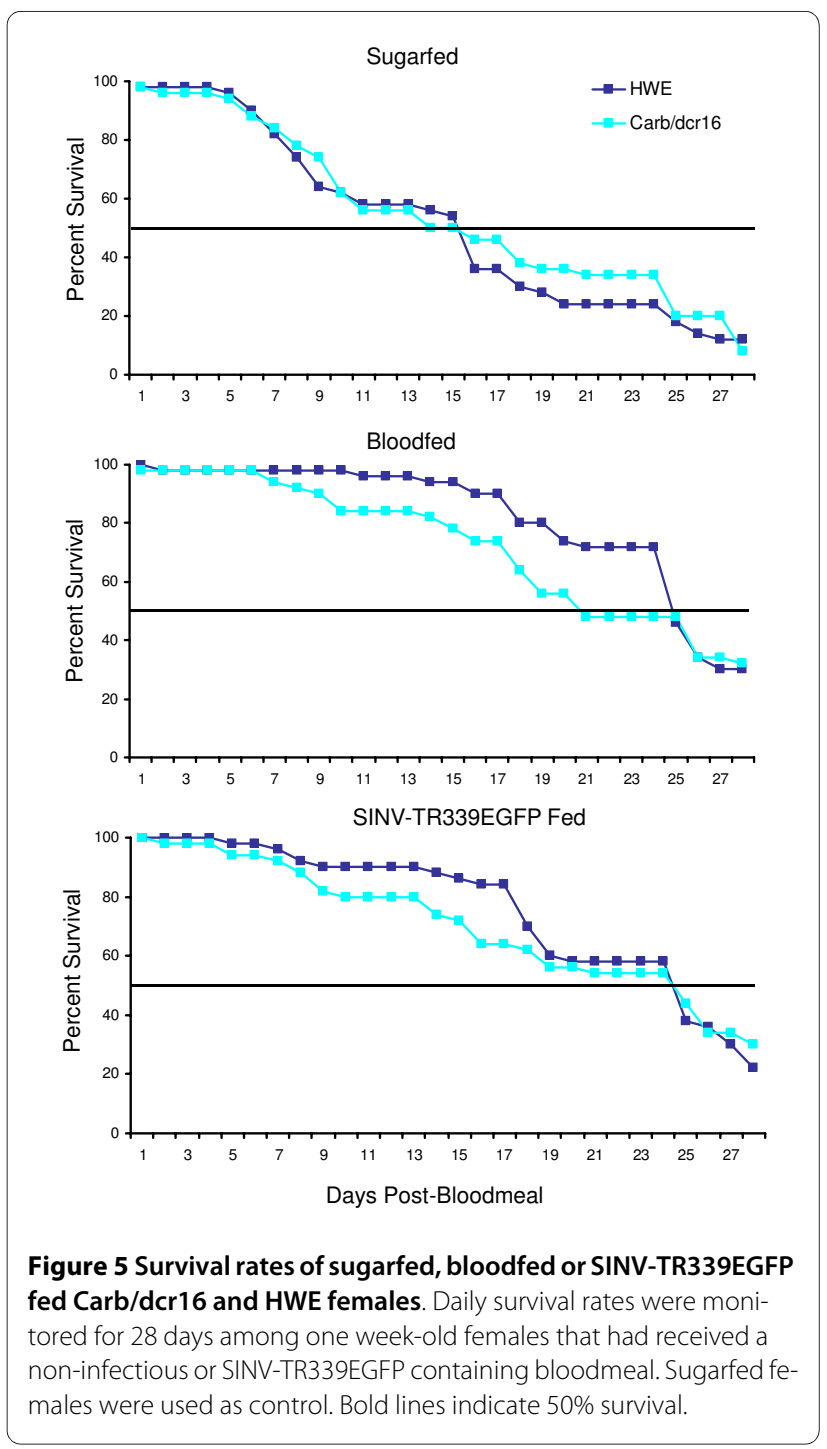

we developed a novel tool to study arbovirus-mosquito interactions at the molecular level. With current genetic tools it is not possible to generate a stable gene-knockout mutant of Ae. aegypti via homologous recombination (A.W.E. Franz, N. Jasinskiene, M.R. Smith, K.E. Olson and A.A. James, unpublished results). In addition, although intrathoracic injection of dsRNA has been shown to be sufficient to manipulate the RNAi pathway in mosquitoes $[2,3,6,24,25]$ the strategy presented here bears several advantages. 1) Injuries caused by intrathoracic injection of dsRNAs are eliminated, preventing non-specific triggering of other immune pathways and/or reduced longevity of the insect. 2) Off-target effects caused by high doses of injected dsRNAs dispersed throughout the mosquito body are avoided. 3) Precise temporal and spatial gene targeting is ensured.

$A a-d c r 2$ acts at the beginning of the initiation phase of the siRNAi pathway by cleaving long dsRNA molecules into $\sim 21$ bp duplexes. With the support of $A a-r 2 d 2$ these 
siRNA duplexes are inserted into the RISC complex [31]. When silencing $A a-d c r 2$ using an IR RNA with sequence homology, we expected $A a-d c r 2$ mRNA levels in the cell to diminish over time, which would result in depletion of dicer2 protein. Eventually, there would be insufficient dicer2 enzyme available to maintain the RNAi pathway in a functional state. Based on the pattern of $A e C P A$ promoter-based expression, impairing of the RNAi pathway was supposed to last for only $36 \mathrm{~h}$ during digestion of the bloodmeal in the midgut. Before the onset of Aa-dcr2 mRNA silencing in midgut cells of Carb/dcr16 females, most likely there were sufficient quantities of dicer2 protein synthesized, which could turn the RNAi mechanism against itself. Possibly during the entire $36 \mathrm{~h}$ period of RNAi silencing certain quantities of functional dicer2 prevailed in the midgut cells so that the pathway was compromised in its efficiency and capacity but never completely shut off. Similar lack of complete inhibition of RNAi was observed before when transiently silencing $d c r 2$ in Drosophila S2 cells [27]. This could explain the pattern of the Aa-dcr2 mRNA expression profiles in Carb/dcr16 females, where the efficiency of Aa-dcr2 mRNA silencing fluctuated over time but its expression was never eliminated. Moreover, infection with SINV resulted in increased $A a-d c r 2$ mRNA accumulation in Carb/dcr16 females, showing that the midgut epithelial cells were still able to mobilize additional dicer2 protein, even though the pathway was impaired in the midgut tissue. Increase in $A a-d c r 2$ mRNA accumulation confirms earlier findings that the TR339 strain of SINV triggers the RNAi pathway in Ae. aegypti [3]. However, no mechanism for $A a-d c r 2$ induction has been described so far. We have no clear explanation as to why at 2 days pbm $A a-$ dcr2 mRNA levels were increased in both HWE and Carb/dcr16 females. We observed that levels of transgenic $A a-d c r 2$ silencing varied considerably between the different transgenic mosquito lines that were initially tested. This could be caused by corresponding variations in $A a-d c r 2$ IR RNA expression levels. Based on previous observations with transgenic mosquitoes expressing a marker gene in midgut tissue (A.W.E. Franz, K.E. Olson, A.A. James, unpublished results), the TE integration site in the genome of the mosquito can strongly affect geneof-interest expression levels.

Even though maximal silencing of $A a-d c r 2$ in midguts of SINV-TR339EGFP infected Carb/dcr16 females appeared to be no more than $\sim 50 \%$, it had profound effects on intensity of infection, midgut infection and dissemination rates of the virus at 7 days $\mathrm{pbm}$. Average virus titers in midguts increased from $1750 \mathrm{pfu} / \mathrm{ml}$ in HWE to $14,000 \mathrm{pfu} / \mathrm{ml}$ in Carb/dcr16 mosquitoes. Accordingly, midgut infection rates increased from 33\% (HWE) to $69 \%$ (Carb/dcr16) and virus dissemination rates from 30\% (HWE) to 60\% (Carb/dcr16). These data suggest that the
RNAi pathway in the mosquito midgut tightly controls SINV infection by modulating its replication. Thus, MIB and MEB for SINV-TR339EGFP in Ae. aegypti were virus dose-dependent and in this way affected by the RNAi pathway. Whereas a virus dose-dependent MEB has been reported for the TR339 strain of SINV, no MIB has been observed for this virus $[9,13]$. Despite the fact that the authors used another mosquito strain in their studies, they also used a non-EGFP expressing virus and higher virus concentrations in their bloodmeals, ranging from $10^{8}-10^{9} \mathrm{pfu} / \mathrm{ml}$. In our study the virus concentrations in bloodmeals ranged from $1.7-2.7 \times 10^{7} \mathrm{pfu} / \mathrm{ml}$. In the presence of a functional RNAi mechanism as in HWE mosquitoes, the lower virus concentration in the bloodmeal was probably approaching the threshold for midgut infection. In the RNAi pathway impaired Carb/dcr16 mosquitoes however, this virus concentration was sufficient to cause productive midgut infections.

Between 7 and 14 days pbm a strong reduction of virus infection intensity was observed in midguts of Carb/ dcr16 mosquitoes, causing a decrease in average SINV titers from 14,000 to $2400 \mathrm{pfu} / \mathrm{ml}$. Such strong reduction of virus infection intensity was not observed in the RNAi pathway competent HWE control. After 7 days pbm the RNAi pathway in Carb/dcr16 mosquitoes was no longer compromised as it was during virus acquisition. It appears that the RNAi mechanism, when functional, down-regulated the unusually high SINV concentration in midguts of the transgenic mosquitoes to levels similar to those of the HWE control. This strongly suggests that the task of the RNAi pathway in the mosquito midgut is to keep arbovirus replication at a level that can be tolerated by the mosquito. Modulation of arbovirus infections in mosquitoes has been reported for several virus-vector combinations and research of the last few years eventually confirmed that the RNAi pathway of the mosquito is a major driving force behind this modulation $[2,3,6,14,16,32]$. Nevertheless, recent studies indicate that other innate immune pathways, such as JAK-STAT and/ or Toll also contribute to the modulation of arbovirus infections in insects [33-37].

Since a proposed role for the RNAi pathway in mosquitoes is to protect the insect from pathogenic effects of replicating arboviruses [4-6], we investigated whether SINV-TR339EGFP causes such effects in HWE or Carb/ dcr16 mosquitoes. Our survival curve data indicate that the initial increase in virus titer in Carb/dcr16 females did not cause obvious pathogenic effects. It needs to be pointed out that after 7 days pbm the RNAi pathway was no longer impaired in midguts of Carb/dcr16 mosquitoes and the intensity of infection was strongly modulated. Thus, the RNAi pathway activation in the transgenic mosquito line could have been similar to that in the control for the latter 21 days of the survival study. Our obser- 
vations confirm those by Campbell and co-workers [3] that transient silencing of the RNAi pathway in $A e$. aegypti did not affect longevity of the mosquitoes for seven days after infection with SINV. However, several authors have described pathological effects caused by alphaviruses in mosquito midguts and salivary glands, claiming that these effects could be virus dose-dependent [38-41]. Moreover, systemic expression of a potent RNAi pathway suppressor via a recombinant SINV severely reduced the survival rate of mosquitoes $[4,5]$. Thus, it might be necessary to knockout the RNAi pathway in the insect to reveal long-term effects of a compromised, antiviral immune pathway on mosquito fitness.

\section{Conclusions}

We generated transgenic mosquitoes that have an impaired RNAi pathway in the midgut following ingestion of a bloodmeal. These mosquitoes, Carb/dcr16, represent a novel tool to study arbovirus-mosquito interactions at the molecular level. Temporal impairment of the RNAi pathway in the midgut epithelium of Carb/ dcr16 mosquitoes significantly increased the infection intensity of SINV-TR339EGFP, thereby allowing the virus to overcome MIB and MEB. Thus, both barriers, which are affected by the endogenous RNAi mechanism, appear to be virus dose-dependent phenomena for this SINV strain in Ae. aegypti. Furthermore, the infection pattern of SINV in Carb/dcr16 females suggests that the RNAi pathway is modulating virus replication in the midgut to prevent the virus from reaching harmful concentrations in the insect. As a consequence, longevity of SINVTR339EGFP infected mosquitoes was similar to that of non-infected ones. Overall, our data confirm that the mosquito midgut is the central organ that determines vector competence for arboviruses.

\section{Future Directions}

Using Carb/dcr16 mosquitoes, we plan to evaluate effects of RNAi pathway impairment in the midgut on infection patterns of dengue and Chikungunya viruses, which are naturally transmitted by this mosquito species.

\section{Methods}

Transgene design and generation of transgenic Ae. aegypti Five hundred base-pair cDNA fragments corresponding to the ribonuclease I domain encoding region of $A a-d c r 2$ were inserted in sense and anti-sense orientations into $p$ SLfa1180fa. Both fragments were separated by the small intron of the Aa-sialonkinin I gene [42]. The resulting inverted-repeat (IR) DNA was placed downstream of the AeCPA promoter and a SV40 transcription termination signal was added at the 3 ' terminus of the IR construct. This construct was then inserted into the non-autonomous Mariner Mos1 TE containing an eye tissue-specific
EGFP expression cassette to allow easy identification of individual mosquitoes harboring the TE [43]. Transgenic mosquitoes were generated as described earlier $[24,44,45]$ using the Higgs White Eye (HWE) strain of $A e$. aegypti as recipient [46]. Mosquitoes received bloodmeals from mice following Colorado State University Institutional Animal Care and Use Committee (IACUC) regulations (IACUC protocol: 09-1365A-01). Mosquitoes were reared in a BSL2 insectary at $28^{\circ} \mathrm{C}$ and $80 \%$ relative humidity. Hemizygous Carb/dcr16 mosquitoes were maintained as an inbred colony. In the experiments intercrossed generations $G_{5}$ to $G_{8}$ were used among which 60 $80 \%$ of the individuals were transgenic based on fluorescent eye marker expression.

\section{Characterization of the transgene integration site}

The transgene integration site in Carb/dcr16 mosquitoes was characterized by Genome Walking using the GenomeWalker Universal Kit and Advantage2 Polymerase (Clontech, Palo Alto, CA) as described before [25]. Genespecific primers for the detection of genomic DNA surrounding the Mariner Mos1 left arm in Carb/dcr16 mosquitoes were maLeft FWD (5'caattatgacgctcaattcgcgcca aac3') and maLeft_nested FWD (5'gtggttcgacagtcaaggttgaca $\mathrm{cttc} 3$ '). To detect genomic DNA surrounding the right arm of the TE primers maRight FWD (5'gcagtttc caatcgcttgcgagagatg3') and maRight_nested FWD (5' atgagttgaacgagaggcagatggagag3') were used.

\section{Detection of transgene expression levels by Northern blot analysis}

Expression of the IR RNA targeting Aa-dcr2 in Carb/ dcr16 mosquitoes was evaluated by Northern blot analysis. Using TRIzol Reagent (Invitrogen, Carlsbad, CA) total RNA was extracted from pools of 120 midguts of transgenic and HWE control females that had received a sugarmeal or bloodmeal 18, 30 or $72 \mathrm{~h}$ before. For each sample $5 \mu \mathrm{g}$ of RNA was separated electrophoretically in a 1.2\% agarose gel and blotted onto a positively charged nylon membrane (Applied Biosystems, Foster City, CA). The blot was hybridized with a random primed $500 \mathrm{bp}$ ${ }^{32} \mathrm{P}$-dCTP labeled cDNA probe $(3000 \mathrm{ci} / \mathrm{mmol})$, which was prepared using the DECAprime II DNA Labeling Kit (Applied Biosystems). The sequence of the probe corresponded to the Aa-dcr2 IR effector of Carb/dcr16 mosquitoes.

\section{Quantification of Aa-dcr2 mRNA levels}

Quantitative reverse transcriptase PCR (qRT-PCR) was conducted to determine $A a-d c r 2$ mRNA levels in midguts of females. Midguts from 20 females were dissected at $1,2,3,4$, and 7 days pbm and stored in TRIzol Reagent (Invitrogen) at $-80^{\circ} \mathrm{C}$ until total RNA was extracted according to the manufacturer's protocol. qRT-PCR was 
performed using the QuantiFast SYBR Green RT-PCR kit (Qiagen, Valencia, CA) and the iQ5 Real-Time PCR Detection System (BioRad, Herciles, CA). To quantify Aa-dcr2 cDNAs, primers dcr2 qFWD (5'tcggaaatt tcaacgatagctcgtaaca3') and dcr2 qREV (aattcgcgtagga accgtactccggatt3') were used. The RT reaction was conducted for $10 \mathrm{~min}$ at $50^{\circ} \mathrm{C}$ followed by a PCR reaction $(5$ min at $95^{\circ} \mathrm{C}$ and 35 cycles of $10 \mathrm{~s}$ at $95^{\circ} \mathrm{C}$ and $30 \mathrm{~s}$ at $60^{\circ} \mathrm{C}$ ). Aa-dcr2 standards consisted of serially diluted cDNA clones containing the $A a-d c r 2$ PCR product (181 bp in size) and were used to derive the copy number per ng of total RNA. Resulting $A a-d c r 2$ copy numbers obtained from midgut RNA of bloodfed or virus-infected females were normalized for copy numbers obtained from midgut RNA of sugarfed females.

\section{Oral infection of Carb/dcr16 and HWE mosquitoes with SINV-TR339EGFP}

Prior to a bloodfeeding experiment mosquitoes were reared on raisins and water. A large $2.5 \mathrm{~L}$ carton typically contained 125 females and 10 males. Raisins and water were removed from the cartons $36 \mathrm{~h}$ and $5 \mathrm{~h}$, respectively before bloodfeeding. To infect females with SINVTR339EGFP one week post-emergence, defibrinated sheep blood was mixed at a 1:1 ratio with virus freshly harvested from Vero cell culture medium. SINV titers in bloodmeals ranged from 1.7 to $2.7 \times 10^{7} \mathrm{pfu} / \mathrm{ml}$. HWE and Carb/dcr 16 females were fed for $1 \mathrm{~h}$ using one glass feeder per carton, which contained $2 \mathrm{ml}$ of bloodmeal maintained at $37^{\circ} \mathrm{C}$. After bloodfeeding, the mosquitoes were sorted for females that were three quarters or fully engorged. These individuals were further reared in 470 $\mathrm{ml}$ cartons (40 females/carton) and fed with sucrose and water until further analysis.

\section{Propagation of SINV-TR339EGFP and determination of virus titers by plaque assay}

SINV-TR339EGFP virus stocks were generated from an infectious cDNA clone that contained the EGFP marker gene under control of a duplicated sub-genomic promoter located upstream of the coding sequence for the structural genes [3]. Virus titers from individual midguts and bodies were determined by plaque assay at 7 and 14 days pbm as described before [2]. Briefly, samples were homogenized in $0.5 \mathrm{ml}$ MEM medium with 7\% FBS and filtered with Acrodisc HT Tuffryn $0.2 \mu \mathrm{m}$ syringe filters (Pall Life Sciences, East Hills, NY). Vero cells were seeded into 24-well plates and left for three days to achieve confluence. Cells were infected with 10 -fold serial dilutions of individual midgut or carcass homogenates. Cells were incubated for $1 \mathrm{~h}$ at $37^{\circ} \mathrm{C}$ before overlaid with an agarosenutrient mixture [1× Medium 199 (Sigma-Aldrich, St. Louis, $\mathrm{MO}$ ), $10 \% \mathrm{FBS}, 4 \% \mathrm{NaHCO}_{3}, 0.5 \%$ MEM vitamins, 0.5\% MEM amino acids (Mediatech Inc., Manassas, VA)].
The plates were incubated at $37^{\circ} \mathrm{C}$ for 4 days. Cells were then stained with MTT (3- [4,5-dimethylthiazol-2-yl]2,5-diphenyltetrazolium bromide) (Sigma-Aldrich, St. Louis, MO), incubated at $37^{\circ} \mathrm{C}$ for $24 \mathrm{~h}$ and the number of plaques was counted for each sample. Virus titers of individual mosquitoes were calculated as $\mathrm{pfu} / \mathrm{ml}$.

\section{Survival curve of Ae. aegypti}

Seven day-old Carb/dcr16 and HWE females were either fed with a non-infectious bloodmeal or with a bloodmeal containing SINV-TR339EGFP. After bloodfeeding, 50 mosquitoes of each treatment were put into $470 \mathrm{ml}$ cardboard containers and provided with sugar and water. A control consisting of females that were sugarfed only was included in the experiment. For a period of 28 days after bloodfeeding the daily number of surviving mosquitoes in each container was recorded.

\section{Statistical analysis}

Statistical analyses were performed using SAS Statistical Analysis Software (SAS Institute Inc., Cary, NC). The MIXED procedure was used for restricted maximum likelihood parameter estimation with incomplete data. Aa-dcr2 ratios and SINV-TR339EGFP infection levels were normalized using a $\log 10$ transformation. Aa- $d c r 2$ ratios, virus infection levels, and virus infection/dissemination rates were then analyzed using the least-squares means test followed by pair-wise comparisons with the Tukey-Kramer test.

\section{Authors' contributions}

CCHK designed and performed the qRT-PCR assays, virus challenge and survival experiments, analyzed the data and wrote the manuscript. JP assisted with sample preparations, qRT-PCR assays, mosquito rearing and virus challenge experiments. ISV performed the Northern blot. KEO conceived the study, analyzed the data and edited the manuscript. AWEF conceived the study, generated the IR effector construct and the transgenic mosquitoes, performed the Genome Walking experiment, analyzed the data and edited the manuscript. All authors read and approved the final manuscript.

\section{Acknowledgements}

We thank J. zumBrunnen for help with statistical analyses, M. Smith for initial mosquito screening, M. Heersink for help with mosquito rearing, and C. Meridith for providing stocks of HWE eggs. This work was funded by R01 grant Al073298-01 from the National Institutes of Health.

\section{Author Details}

Arthropod-Borne and Infectious Diseases Laboratory, Department of Microbiology, Immunology and Pathology, Colorado State University, Fort Collins, CO 80523 USA

Received: 22 December 2009 Accepted: 28 April 2010

Published: 28 April 2010

\section{References}

1. Bosher JM, Labouesse M: RNA interference: genetic wand and genetic watchdog. Nature Cell Biol 2000, 2:31-36.

2. Keene KM, Foy BD, Sanchez-Vargas I, Beaty BJ, Blair CD, Olson KE: RNA interference acts as a natural antiviral response to O'nyong-nyong virus (Alphavirus; Togaviridae) infection of Anopheles gambiae. P Natl Acad Sci USA 2004, 101:17240-17245. 
3. Campbell CL, Keene KM, Brackney DE, Olson KE, Blair CD, Wilusz J, Foy BD: Aedes aegypti uses RNA interference in defense against Sindbis virus infection. BMC Microbiol 2008, 8:47.

4. Cirimotich CM, Scott JC, Phillips AT, Geiss BJ, Olson KE: Suppression of RNA interference increases alphavirus replication and virus-associated mortality in Aedes aegypti mosquitoes. BMC Microbio/ 2009, 9:49.

5. Myles KM, Wiley MR, Morazzani EM, Adelman ZN: Alphavirus-derived small RNAs modulate pathogenesis in disease vector mosquitoes. $P$ Natl Acad Sci USA 2008, 105:19938-19943.

6. Sanchez-Vargas I, Scott JC, Poole-Smith BK, Franz AWE, Barbosa-Solomieu $\checkmark$, Wilusz J, Olson KE, Blair CD: Dengue virus type 2 infections of Aedes aegypti are modulated by the mosquito's RNA interference pathway. PLOS Pathog 2009, 5:e1000299.

7. Uchil PD, Satchidanandam V: Architecture of the flavivirus replication complex. Protease, nuclease, and detergents reveal encasement within double-layered membrane compartments. J Biol Chem 2003, 278:24388-24398

8. Medlock JM, Snow KR, Leach S: Possible ecology and epidemiology of medically important mosquito-borne arboviruses in Great Britain. Epidemiol and Infect 2006, 135:466-482.

9. Myles KM, Pierro DJ, Olson KE: Comparison of the transmission potential of two genetically distinct Sindbis viruses after oral infection of Aedes aegypti (Diptera: Culicidae). J Med Entomol 2004, 41:95-106.

10. Taylor RM, Hurlbut HS, Work TH, Kingston JR, Frothingham TE: Sindbis virus: A newly recognized arthropod-transmitted virus. Am J Trop Med Hyg 1955, 4:844-862.

11. McKnight KL, Simpson DA, Lin S, Knott TA, Polo JM, Pence DF, Johannsen DB, Heidner HW, Davis NL, Johnston RE: Deduced consensus sequence of Sindbis virus strain AR339: Mutations contained in laboratory strains which affect cell culture and in vivo phenotypes. J Virol 1996, 70:1981-1989.

12. Klimstra WB, Ryman KD, Johnston RE: Adaptation of Sindbis virus to BHK cells selects for use of heparin sulfate as an attachment receptor. J Virol 1998, 72:7357-7366.

13. Pierro DJ, Powers EL, Olson KE: Genetic determinants of Sindbis virus strain TR339 affecting midgut infection in the mosquito Aedes aegypti. J Gen Virol 2007, 88:1545-1554

14. Hardy JL, Houk EJ, Kramer LD, Reeves WC: Intrinsic factors affecting vector competence of mosquitoes for arboviruses. Annu Rev Entomol 1983, 28:229-262

15. Mellor PS: Replication of arboviruses in insect vectors. J Comp Pathol 2000, 123:231-247.

16. Kramer LD, Hardy JL, Presser SB, Houk EJ: Dissemination barriers for western equine encephalomyelitis virus in Culex tarsalis infected after digestion of low viral doses. Am J Trop Med Hyg 1981, 30:190-197.

17. Seabaugh RC, Olson KE, Higgs S, Carlson JO, Beaty BJ: Development of a chimeric sindbis virus with enhanced per os infection of Aedes aegypti. Virology 1998, 243:99-112.

18. Miller BR, Mitchell CJ: Genetic selection of a flavivirus-refractory strain of the yellow fever mosquito Aedes aegypti. Am J Trop Med Hyg 1991, 45:399-407.

19. Bosio CF, Beaty BJ, Black WC: Quantitative genetics of vector competence for dengue-2 virus in Aedes aegypti. Am J Trop Med Hyg 1998, 59:965-970

20. Weaver SC, Scherer WF, Cupp EW, Castello DA: Barriers to dissemination of Venezuelan encephalitis viruses in the Middle American enzootic vector mosquito, Culex (Melanoconion) taeniopus. Am J Trop Med Hyg 1984, 33:953-960

21. Bernhardt SA: Aedes aegypti and dengue virus investigation of anatomic, genomic, and molecular determinants of vector competence. In PhD thesis Colorado State University, Department of Microbiology, Immunology and Pathology; 2009.

22. Edwards MJ, Moskalyk LA, Donelly-Doman M, Vlaskova M, Noriega FG, Walker VK, Jacobs-Lorena M: Characterization of a carboxypeptidase A gene from the mosquito, Aedes aegypti. Insect Mol Biol 2000, 9:33-38.

23. Moreira L, Edwards MJ, Adhami F, Jasinskiene N, James AA, Jacobs-Lorena M: Robust gut-specific gene expression in transgenic Aedes aegypti mosquitoes. P Natl Acad Sci USA 2000, 97:10895-10898.

24. Franz AWE, Sanchez-Vargas I, Adelman ZN, Blair CD, Beaty BJ, James AA, Olson KE: Engineering RNA interference-based resistance to dengue virus type 2 in genetically modified Aedes aegypti. P Natl Acad Sci USA 2006, 103:4198-4203.
25. Franz AWE, Sanchez-Vargas I, Piper J, Smith MR, Khoo CCH, James AA, Olson KE: Stability and loss of a virus resistance phenotype over time in transgenic mosquitoes harbouring an antiviral effector gene. Insect Mol Biol 2009, 18:661-672.

26. Adelman ZN, Anderson MA, Morazzani EM, Myles KM: A transgenic sensor strain for monitoring the RNAi pathway in the yellow fever mosquito, Aedes aegypti. Insect Biochem Mol Biol 2008, 38:705-713.

27. Bernstein E, Caudy AA, Hammond SM, Hannon GJ: Role for a bidentate ribonuclease in the initiation step of RNA interference. Nature 2001, 409:363-366.

28. Hoa NT, Keene KM, Olson KE, Zheng L: Characterization of RNA interference in an Anopheles gambiae cell line. Insect Biochem Mol Biol 2005, 33:949-957.

29. Coates CJ, Jasinskiene N, Miyashiro L, James AA: Mariner transposition and transformation of the yellow fever mosquito, Aedes aegypti. P Natl Acad SciUSA 1998, 95:3748-3751.

30. Bian G, Shin SW, Cheon HM, Kokoza V, Raikhel AS: Transgenic alteration of toll immune pathway in the female mosquito Aedes aegypti. P Natl Acad Sci USA 2006, 102:13568-13573.

31. Kim K, Lee YS, Harris D, Nakahara K, Carthew RW: The RNAi pathway initiated by Dicer-2 in Drosophila. Cold SH Q B 2006, 71:39-44.

32. Murphy FA: Cellular resistance to arbovirus infection. Ann NY Acad Sci 1975, 266:197-203.

33. Dostert C, Jouanguy E, Irving P, Troxler L, Galiana-Arnoux HC, Hoffmann JA, Imler J: The Jak-STAT signaling pathway is required but not sufficient for the antiviral response of drosophila. Nature Immunol 2005, 6:946-953.

34. Zambon RA, Nandakumar M, Vakharia VN, Wu LP: The toll pathway is important for an antiviral response in Drosophila. P Natl Acad Sci USA 2005, 102:7257-7262.

35. Sanders HR, Foy BD, Evans AM, Ross LS, Beaty BJ, Olson KE, Gill SS: Sindbis virus induces transport processes and alters expression of innate immunity pathway genes in the midgut of the disease vector, Aedes aegypti. Insect Biochem Mol Biol 2005, 35:1293-1307.

36. Xi Z, Ramirez JL, Dimopoulos G: The Aedes aegypti Toll pathway controls dengue virus infection. PLOS Pathog 2008, 4:e1000098.

37. Souza-Neto JA, Sim S, Dimopoulos G: An evolutionary conserved function of the JAK-STAT pathway in anti-dengue defense. $P$ Natl Acad SCiUSA 2009, 106:17841-17846.

38. Mims CA, Day MF, Marshall ID: Cytopathic effect of semliki forest virus in the mosquito Aedes aegypti. Am J Trop Med Hyg 1966, 16:775-784.

39. Weaver SC, Scott TW, Lorenz LH, Lerdthusnee K, Romoser WS: Togavirusassociated pathologic changes in the midgut of a natural mosquito vector. J Virol 1988, 62:2083-2090

40. Cooper LA, Sina BJ, Turell MJ, Scott TW: Effects of initial dose on eastern equine encephalomyelitis virus dependent mortality in intrathoracically inoculated Culiseta melanura (Diptera: Culicidae). Med Entomol 2000, 37:815-819.

41. Bowers DF, Coleman CG, Brown DT: Sindbis virus-associated pathology in Aedes albopictus. J Med Entomol 2006, 40:698-705.

42. Beerntsen BT, Champagne DE, Coleman JL, Campos YA, James AA Characterization of the Sialokinin I gene encoding the salivary vasodilator of the yellow fever mosquito, Aedes aegypti. Insect Mol Biol 1999, 8:459-467.

43. Horn C, Jaunich B, Wimmer EA: Highly sensitive, fluorescent transformation marker for Drosophila transgenesis. Dev Genes Evol 2000, 210:623-629.

44. Jasinskiene N, Coates CJ, Benedict MQ, Cornel AJ, Salazar-Rafferty C, James AA, Collins FH: Stable transformation of the yellow fever mosquito, Aedes aegypti, with the Hermes element from the housefly. P Natl Acad Sci USA 1998, 95:3743-3747.

45. Jasinskiene N, Juhn J, James AA: Microinjection of A. aegypti embryos to obtain transgenic mosquitoes. J Visual Exp 2007, 5:219.

46. Wendell MD, Wilson TG, Higgs S, Black WC: Chemical and gamma-ray mutagenesis if the white gene in Aedes aegypti. Insect Mol Biol 2000, 9:119-125.

doi: 10.1186/1471-2180-10-130

Cite this article as: Khoo et al., The RNA interference pathway affects midgut infection- and escape barriers for Sindbis virus in Aedes aegypti BMC Microbiology 2010, 10:130 